\title{
ORIGINAL ARTICLE \\ Rasch measurement properties of the Pain Medication Questionnaire in persons with spinal cord injury
}

\author{
BN Hand, CA Velozo and JS Krause
}

Study design: Secondary analysis of cross-sectional population-based self-report data.

Objective: To determine how well the Pain Medication Questionnaire (PMQ) measures risk of pain medication misuse and its precision in separating individuals with spinal cord injury (SCI) into meaningful classification categories.

Setting: Academic medical center in Southeastern United States.

Methods: Data were collected from a population-based registry of $\mathrm{SCl}(n=971)$. Eligible participants included adults with traumatic $\mathrm{SCl}$ with residual effects who were at least 1 year post injury and 18 years of age and who had PMQ data in which they reported active use of pain medication at the time of the study $(n=745)$.

Results: Most items (23/26) of the PMQ contributed to a single unidimensional construct. Rasch analysis results revealed that the rating scale, majority of persons ( $>93 \%$ ), and majority of items (20/23) fit the Rasch measurement model. The PMQ demonstrated adequate reliability (person reliability $=0.67$ ) and separated persons into two strata-those likely to misuse pain medication and those with low liklihood of misusing pain medication.

Conclusions: Findings offer a deeper understanding of the measurement properties of the $P M Q$ as a precursor for widespread population-based studies to elucidate the incidence of pain medication misuse in persons with SCl. Results also have important research and clinical implications for commonly used PMQ total score cut-offs, which may misclassify an individual's risk of pain medication misuse.

Spinal Cord (2017) 55, 1117-1122; doi:10.1038/sc.2017.89; published online 1 August 2017

\section{INTRODUCTION}

Up to $60 \%$ of persons with spinal cord injury (SCI) experience pain. ${ }^{1}$ Chronic pain after SCI has been associated with depressive symptoms, anxiety, poorer quality of life, and sleep disturbances. ${ }^{2,3}$ Individuals with SCI report the greatest pain relief from opioid medications, and $82 \%$ report taking at least one prescription medication for pain management. ${ }^{4}$ Given the widespread implications of pain post-SCI, and the epidemic of pain medication overdose in the general population, ${ }^{5}$ it is critical that clinicians working with individuals with SCI have a thorough understanding of the potential for pain medication misuse (PMM) as well as valid and reliable means of identifying individuals at elevated risk of PMM.

PMM has been documented in $17-25 \%$ of persons with SCI. ${ }^{6,7}$ Various factors have been associated with increased risk for PMM in individuals with SCI including: greater reported pain intensity; more limitations in daily activities due to pain; higher frequency of pain medication use; smoking tobacco; cannabis use; anxiety; depressive symptoms; and impulsive sensation-seeking. ${ }^{6,7}$ PMM is an important issue for clinical consideration, as it has been associated with higher likelihood of fall-related injuries in ambulatory persons with SCI, ${ }^{8}$ as well as fractures, overdose, and myocardial infarction in the general population. ${ }^{9}$ Given that cardiovascular disease is one of the leading causes of mortality ${ }^{10}$ and fractures are associated with increased risk of mortality ${ }^{11}$ in individuals with SCI, PMM in this population should be carefully monitored.
One questionnaire utilized for identifying individuals at risk for PMM is the Pain Medication Questionnaire (PMQ). ${ }^{12}$ The PMQ consists of 26 items rated on a five-point Likert scale with total scores ranging from 0-104, where higher scores indicate higher risk of PMM. Initial psychometric testing on the PMQ showed moderate, but acceptable, reliability coefficients (test-retest reliability Pearson's $r=0.85$, Cronbach's $\alpha=0.73$ ) as well as correlation of scores with levels of psychosocial distress (Pearson's $r=0.23-0.35, P<0.05$ ) and physical functioning (Pearson's $r=0.23-0.36, P<0.01$ ). ${ }^{12}$ PMQ scores have also demonstrated sensitivity to change over time and have been shown to be predictive of future pain-medication seeking behaviors and early termination from pain medication treatment programs. ${ }^{13,14}$

Previous research studies have utilized cutoff values for PMQ total scores $^{6,7}$ or have divided total scores into tertiles ${ }^{14,15}$ to identify individuals who demonstrate behaviors predictive of PMM. The difficulty with approaches based on total scores is twofold: (1) they assume that the PMQ measures a single, unidimensional construct, and (2) they assume an interval-level scale, which is a prerequisite for additivity of scores. ${ }^{16}$ There is some literature to suggest that the PMQ is multidimensional; previous psychometric testing on the PMQ revealed items with low point-total correlations ${ }^{15}$ and 10 significant components were identified via a principal component analysis (PCA). ${ }^{17}$ Additionally, it is known that ordinal-level observations, such as those obtained from the rating scale of the PMQ, do not sufficiently approximate interval-level data and, thus, are not sufficient 


\section{Table 1 Participant characteristics}

\begin{tabular}{lr}
\hline Mean age (s.d.) & $50.5(16.3 .0$ \\
Mean years since injury (s.d.) & \\
Gender & $71.9 \%$ \\
Male $(n=536)$ & $28.1 \%$ \\
Female $(n=209)$ & \\
& \\
Race/ethnicity & $56.6 \%$ \\
White non-Hispanic $(n=422)$ & $35.2 \%$ \\
Black non-Hispanic $(n=262)$ & $2.0 \%$ \\
Hispanic $(n=15)$ & $3.1 \%$ \\
Other non-Hispanic $(n=23)$ & \\
Injury level & \\
C1-C4 $(n=197)$ & $30.4 \%$ \\
C5-C8 ( $n=203)$ & $31.4 \%$ \\
Non-cervical $(n=247)$ & $38.2 \%$ \\
Ambulation status & \\
C1-C4 non-ambulatory $(n=34)$ & \\
C5-C8 non-ambulatory $(n=59)$ & $5.8 \%$ \\
Non-cervical non-ambulatory $(n=94)$ & $10.1 \%$ \\
All levels ambulatory $(n=396)$ & $67.9 \%$
\end{tabular}

For some variables, there were a limited amount of missing data. The number of responses in each category are given, but the percentages in the table have been adjusted for nonrespondents.

for the generation of summed total scores. ${ }^{18}$ As such, it is imperative that the precision of the PMQ for separating individuals into meaningful classification categories be empirically tested utilizing modern measurement techniques, which can transform ordinal scale observations into interval scale measurements.

One such modern measurement technique is Rasch analysis, which allows for direct comparison of item difficulty and person ability on a common, interval-level scale. ${ }^{19}$ Rasch analysis also allows for the examination of a measure's capacity for distinguishing statistically distinct levels of person ability on the measured construct, ${ }^{20}$ making this analytical approach ideal for evaluating the PMQ's capacity to distinguish individuals with and without high likelihood of PMM. In addition, the item and person parameters obtained from Rasch analysis are invariant, that is, sample independent. ${ }^{21}$ Therefore, PMQ measurement properties obtained from a given sample of persons with SCI would remain consistent had a different sample been selected from the population of persons with SCI. This useful feature of Rasch analysis maximizes the generalizability of sample findings to the broader clinical population of individuals with SCI.

\section{Purpose}

The objective of the present study is to determine how well the PMQ measures the construct of risk for PMM and its precision in separating individuals with SCI into meaningful classification categories that indicate likelihood of PMM.

\section{MATERIALS AND METHODS}

\section{Data source}

The present study utilized a secondary analysis approach of data collected from the South Carolina SCI Surveillance System Registry (SCISSR), an annual population-based registry of SCI. Incident cases of SCI were identified using International Classification of Diseases, 9th Revision, Clinical Modification codes of $806(0.0-0.9)$ and $952(0.0-0.9)$. Personal identifiers were used to eliminate duplicate admissions, and nonresidents of South Carolina were excluded. Persons with SCI and hospital discharge in 1998 to 2012 were eligible for the SCISSR if at the time of the study they: (a) were $\geqslant 18$ years of age; (b) were $\geqslant 1$ year post injury; and (c) had traumatic SCI with residual effects. Data on diagnoses in the SCISSR were verified through random selection of medical charts. ${ }^{22}$

The SCISSR was used as a basis for identifying participants for a more detailed follow-up self-report assessment, collected by mail. There were 971 participants in the follow-up. The present analysis included PMQ data for individuals in the follow-up who reported active use of pain medication at the time of the data collection $(n=745)$. Participants were an average of seven years post-SCI and 50.5 years of age (Table 1). Seventy-one percent of participants were male. A majority of participants were white non-Hispanic $(n=422)$ or black non-Hispanic $(n=262)$, had a non-cervical SCI $(n=247)$, and were ambulatory $(n=396)$. As with all self-report, there were varying degrees of missing data on individual items.

\section{Analyses}

Tests of unidimensionality. As unidimensionality is a key assumption of Rasch measurement, the unidimensionality of the PMQ was explored via exploratory factor analysis (EFA) and PCA of Rasch-derived residuals. An EFA was conducted with principal factors estimation and oblique rotation in the statistical software $\mathrm{R}$ with package 'psych. ${ }^{23}$ A polychoric correlation matrix was generated for only those participants with complete data $(n=646)$, due to the ordinal nature of the data ${ }^{24}$ and used as the input matrix for the EFA. A minimum factor loading of 0.32 was chosen as the level of significance, as this equates to approximately $10 \%$ overlapping variance with other items in that factor. ${ }^{25}$

In addition, PCA of Rasch-derived residuals was conducted based on the results of the EFA. All items that loaded 0.32 or above on the EFA were included in the PCA. The following three criteria were used to examine results for unidimensionality of included items: 1) the eigenvalue of the first residual component, after the Rasch-derived construct is removed, is $\leqslant 2.0 ; 26$ 2) the magnitude of the item loadings on the first residual component are $<\left.|0.38|\right|^{27}$ and 3 ) item infit and outfit statistics are $<2.0$. The impact of multidimensionality on the estimation of person measures was evaluated by conducting a series of independent $t$-tests and constructing $95 \%$ confidence intervals in a plot of person measures derived from all items and subsets of items identified as contributing to multidimensionality. ${ }^{27}$ The PCA of Rasch residuals was conducted in WINSTEPS software version 3.90. $0^{28}$ and included participants with and without missing data $(n=745)$. The overall missing data rate for the 26 original PMQ items was $2.10 \%$. During WINSTEPS estimation, the observed marginal counts and the observed and expected marginal scores are computed from non-missing observations. ${ }^{26}$

Rasch analysis. Once a reasonably unidimensional set of items was identified from the PMQ, a rating scale model Rasch analysis with JML estimation was conducted using WINSTEPS, version 3.90.0. ${ }^{28}$ First, the appropriateness of the rating scale was evaluated using the following criteria: (1) at least 10 observations of each category, collapsed across all items; (2) monotonicity of rating scale categories (that is, $0-4$ ) as evidenced by an increase in average category difficulty with increasing category value; and (3) outfit mean-square is $<2.0$. Second, the fit of the items and persons to the Rasch model was evaluated by examining infit and outfit mean squares and standardized $z$ values. ${ }^{29}$ Mean square values $>1.70$, as well as standardized $z$-values greater than 2.0 were considered indicative of misfit to the Rasch model. ${ }^{30}$ Third, reliability indicators were examined including: (1) person reliability, which represents the reproducibility of person ordering and was interpreted such that values $\geqslant 0.5$ were considered adequate, $\geqslant 0.80$ were considered good, and $\geqslant 0.90$ were considered high, ${ }^{26}$ and (2) the separation index, which was used to calculate the number of statistically distinct ability strata in the sample. ${ }^{20}$ The number of person strata is calculated according to the formula $\frac{(4 G+1)}{3}$, where $G$ is the person separation index and is an indicator of the number of statistically distinct person measures with centers three calibration errors apart. ${ }^{20}$ Test targeting, test coverage, and item hierarchy were examined visually using person-item maps. Last, we examined differential item functioning (DIF) of included PMQ items for individuals with cervical vs non-cervical SCI using the Mantel test. ${ }^{26}$ The rationale behind this analysis is that some studies have shown 
Table 2 Loadings from exploratory factor analysis (EFA) and principal component analysis (PCA) of standardized Rasch residuals

\begin{tabular}{|c|c|c|c|c|}
\hline Item & $\begin{array}{c}\text { EFA } \\
\text { Loading }^{\text {a }}\end{array}$ & $\begin{array}{c}\text { PCA } \\
\text { Loading }\end{array}$ & $\begin{array}{c}\text { Infit } \\
\mathrm{MnSq}\end{array}$ & $\begin{array}{l}\text { Outfit } \\
\text { MnSq }\end{array}$ \\
\hline Believe not enough ${ }^{a}$ & 0.55 & 0.54 & 0.86 & 0.99 \\
\hline Feel better higher dose & 0.56 & 0.52 & 0.92 & 0.91 \\
\hline Difficulty getting meds & 0.60 & 0.49 & 10.04 & 0.94 \\
\hline Dr does not spend time ${ }^{a}$ & 0.47 & 0.47 & 0.98 & 0.96 \\
\hline Times request higher dose & 0.63 & 0.28 & 0.72 & 0.63 \\
\hline Borrow meds & 0.70 & 0.22 & 0.96 & 0.60 \\
\hline Visit emergency room for pain & 0.57 & 0.20 & 0.97 & 0.77 \\
\hline Times early refill & 0.72 & 0.16 & 0.92 & 0.72 \\
\hline Take more than prescribed & 0.77 & 0.13 & 0.59 & 0.48 \\
\hline Drink to control pain & 0.37 & 0.09 & 1.09 & 0.95 \\
\hline Meds from > $1 \mathrm{Dr}$ & 0.60 & 0.08 & 1.64 & 0.99 \\
\hline Run out early & 0.72 & 0.07 & 0.79 & 0.65 \\
\hline Family obtains meds & 0.70 & 0.03 & 1.69 & 0.70 \\
\hline Difficulty thinking clearly & 0.44 & -0.47 & 0.91 & 0.91 \\
\hline Nausea/constipation & 0.35 & -0.46 & 0.98 & 1.15 \\
\hline Take when anxious & 0.47 & -0.41 & 0.94 & 0.95 \\
\hline I think I'm dependent & 0.52 & -0.37 & 0.97 & 0.91 \\
\hline Save unused for later & 0.43 & -0.32 & 1.17 & 1.35 \\
\hline Family thinks dependent & 0.49 & -0.30 & 1.20 & 1.36 \\
\hline Would mind quitting & -0.32 & -0.28 & 2.09 & 2.75 \\
\hline Number conditions & 0.34 & -0.17 & 1.07 & 1.09 \\
\hline Times misplaced & 0.59 & -0.07 & 1.11 & 0.72 \\
\hline Take sedatives & 0.68 & -0.05 & 0.97 & 0.75 \\
\hline Helpful to call Dr & 0.30 & - & - & - \\
\hline Preference about med type & 0.03 & - & - & - \\
\hline
\end{tabular}

Abbreviations: Dr, doctor; MnSq, mean square.

aExploratory factor analysis loadings of all items on Factor 1. Items that also had significant factor loadings on Factor 2 are identified with a superscript 'a.' Items with non-significant (that is, $<0.32$ ) loadings were not included in the principal component analysis.

that individuals with cervical SCI experience higher levels of pain than persons with non-cervical SCI. ${ }^{31,32}$ Thus, it is important to assess whether the measurement properties of the PMQ are consistent across these diagnostic populations. DIF contrasts, which represent the maginitude of the difference in item difficulties between classification groups, $\geqslant 0.43$ logits were interpreted as slight to moderate and $\geqslant 0.64$ logits were interpreted as moderate to large. ${ }^{26}$ Problematic DIF was identified by statistically significant $\chi^{2}$ test and DIF contrast $\geqslant 0.43$ logits.

\section{Statement of ethics}

We certify that all applicable institutional and governmental regulations concerning the ethical use of human volunteers were followed during the course of this research.

\section{RESULTS}

\section{Unidimensionality}

Exploratory factor analysis. Results of the EFA suggest that a twofactor solution provided the optimum explanation of the observed data. Review of the factor loadings (Table 2) revealed that 23 items had significant loadings $(>0.32)$ on the first factor, and two items had significant loadings on the second factor. Further inspection revealed the two items with significant loadings on the second factor (Item 1: 'I believe I am [NOT] receiving enough medication to relieve my pain,' and Item 2: 'My doctor [DOES NOT] spend enough time talking to me about my pain medication during appointments') also loaded significantly on the first factor. These results were interpreted to be preliminary evidence of unidimensionality for the 23 items that significantly loaded on the first factor. Factor 2 was excluded from subsequent analyses for the following reasons: (1) the two items comprising this factor also loaded significantly on the first factor; and (2) Factor 2 was judged to be insufficient for measurement of a separate construct, due to having only two items.

PCA of Rasch-derived residuals. Using these 23 items, results of PCA of Rasch-derived residuals revealed that the items did not meet our a-priori specified criteria for unidimensionality. Specifically, the first residual component had an eigenvalue of 2.29 , and multiple items had loadings $>10.38 \mid$ on residual contrasts (Table 2). Additionally, one item had an outfit statistic $>2.0$. As a result, the 23 items were subjected to a series of analyses to test the effect of any multidimensionality on person measures. ${ }^{27}$ Specifically, we conducted a series of independent $t$-tests to calculate differences between person measures obtained from the 23-item PMQ when compared with person measures obtained from the items that loaded significantly on the first residual contrast (first seven items listed in Table 2). The rationale behind this approach is that, if the 23-items from the PMQ are sufficiently unidimensional, there will not be a significant distortion in person measures obtained from any subset of these 23 items. Findings revealed that only $1.7 \%$ (95\% binomial confidence interval: $0.8-2.7 \%$ ) of person measures were distorted, which suggests that the subset of 23 PMQ items was sufficiently unidimensional.

\section{Rasch analysis}

Given a set of 23 sufficiently unidimensional items, we proceeded with Rasch analysis and examined indices of fit. Examination of the rating scale indicated acceptable fit (infit and outfit $<2.0$ ) and monotonicity of all rating scale categories. All but three items, which are indicated with asterisks in Table 3, demonstrated adequate fit to the Rasch model as evidenced by infit and outfit statistics $<1.60$ and standardized residuals (standardized $z$-values) $<2.0$. Examination of person fit statistics, using the same criteria, revealed that 47 persons $(6.31 \%)$ demonstrated significant misfit to the model.

The Cronbach's $\alpha$ coefficient for the PMQ was 0.78 and person reliability was adequate (0.67). Additionally, results revealed a person separation index of 1.44, which was input into the formula for strata calculation. ${ }^{20}$ Strata calculation revealed that the analyzed subset of PMQ items functions to separate persons into 2 groups ( strata $=2.25$ ) - those more likely to misuse pain medication and those with low liklihood of misusing pain medication.

Test targeting and coverage, as well as item hierarchy, were examined by inspecting the distribution of the item and person measures from item-person maps. The mean person measure was 1.08 logits (standard error $=0.26$ ) lower than the mean item measure, which suggests the present sample was skewed towards low likelihood of PMM (Figure 1). Floor effects were minimal with $0.7 \%$ of persons who achieved minimum scores; no ceiling effects were found. Personitem maps (Figure 1) and examination of item measures (Table 3) also revealed trends in item hierarchy such that the three easiest items to endorse ('How many painful conditions do you have?'; 'I would feel better with a higher dose of my pain medication;' and 'I believe I am (NOT) receiving enough medication to relieve my pain') are related to thoughts or beliefs about pain medication, while the three items that were least likely to be endorsed ('To help me out, family members have obtained pain medications for me from their own doctors;' 'I get pain medication from more than one doctor in order to have enough medication for my pain;' and 'How many times in the past year have you accidentally misplaced your prescription for pain medication and had to ask for another?') address behaviors which go beyond traditional methods of obtaining pain medication to address pain. Examination of Rasch-half-point threshold maps and items with mean 
Table 3 Measures and fit statistics for 23 items from the Pain Medication Questionnaire

\begin{tabular}{|c|c|c|c|c|c|c|}
\hline Item & Measure & s.e. & Infit $M n S q$ & Infit Zstd & Outfit $M n S q$ & Outfit Zstd \\
\hline Family obtains meds ${ }^{a}$ & 1.96 & 0.17 & 1.69 & 2.3 & 0.70 & -1.1 \\
\hline Meds from $>1$ doctor $^{a}$ & 1.44 & 0.12 & 1.64 & 2.8 & 0.99 & 0.0 \\
\hline Times misplaced & 0.97 & 0.08 & 1.11 & 0.8 & 0.72 & -1.6 \\
\hline Borrow meds & 0.54 & 0.06 & 0.96 & -0.3 & 0.60 & -3.1 \\
\hline Take sedatives & 0.42 & 0.06 & 0.97 & -0.3 & 0.75 & -2.0 \\
\hline Drink to control pain & 0.36 & 0.05 & 1.09 & 0.9 & 0.95 & -0.3 \\
\hline I think I'm dependent & 0.33 & 0.05 & 0.97 & -0.3 & 0.91 & -0.7 \\
\hline Visit emergency room for pain & 0.29 & 0.05 & 0.97 & -0.3 & 0.77 & -1.9 \\
\hline Run out early & 0.14 & 0.04 & 0.79 & -2.7 & 0.65 & -3.4 \\
\hline Times early refill & 0.07 & 0.04 & 0.92 & -1.1 & 0.72 & -2.8 \\
\hline Difficulty thinking clearly & 0.06 & 0.04 & 0.91 & -1.2 & 0.91 & -0.8 \\
\hline Family thinks dependent & -0.06 & 0.04 & 1.20 & 2.7 & 1.36 & 3.3 \\
\hline Times request higher dose & -0.10 & 0.04 & 0.72 & -4.7 & 0.63 & -4.4 \\
\hline Take more than prescribed & -0.13 & 0.04 & 0.59 & -7.5 & 0.48 & -6.8 \\
\hline Take when anxious & -0.20 & 0.04 & 0.94 & -1.0 & 0.95 & -0.6 \\
\hline Nausea/constipation & -0.38 & 0.03 & 0.98 & -0.3 & 1.15 & 1.8 \\
\hline Dr. does not spend time & -0.46 & 0.03 & 0.98 & -0.4 & 0.96 & -0.6 \\
\hline Save unused for later & -0.52 & 0.03 & 1.17 & 3.4 & 1.35 & 4.5 \\
\hline Difficulty getting meds & -0.62 & 0.03 & 1.04 & 1.0 & 0.94 & -1.0 \\
\hline Would mind quittinga & -0.82 & 0.03 & 2.09 & 9.9 & 2.75 & 9.9 \\
\hline Feel better higher dose & -0.86 & 0.03 & 0.86 & -3.6 & 0.99 & -0.1 \\
\hline Believe not enough & -0.98 & 0.03 & 0.92 & -2.1 & 0.91 & -1.8 \\
\hline Number conditions & -1.44 & 0.03 & 1.07 & 1.6 & 1.09 & 1.8 \\
\hline
\end{tabular}

Abbreviations: MnSq, mean square; Zstd, standardized Z-value.

altems with significant misfit.

measures within two standard errors of one another revealed a considerable degree of overlap in the middle of the scale, indicating that item reduction may be possible.

In a post hoc analysis, due to the finding that the PMQ separated persons with SCI into two strata, we examined commonly used total score cutoffs of 25 and $30^{6,7}$ in relation to the item difficulty hierarchies produced by Rash analysis. WINSTEPS software provides complete score-to-measure conversion tables and an ogive curve, which allows for the comparison of total scores on an instrument to logit measures (Figure 2). To allow for direct comparison of these conventional total score cut-offs with Rasch results, we conducted a Rasch analysis with all 26 items from the original PMQ included. Results revealed that the cutoff scores of 25 and 30 were associated with Rasch measures of -0.77 and -0.62 , respectively. Examination of these values on the person-item map (Figure 1) revealed that these scores were: a) near the center of the person measure distribution in the present sample, and b) around the same level as the mean measure of the item: 'I believe I am [NOT] receiving enough medication to relieve my pain.' This suggests that use of these cutoff scores would identify likely pain medication misusers as persons who endorse that item or any items of greater difficulty.

Last, we examined item DIF (Table 4) for individuals with cervical vs non-cerivcal SCI. Participants who did not report their SCI injury level in the survey $(n=96)$ were not included in this analysis. A Bonferonni correction was applied due to multiple comparisons, which resulted in a threshold of significance at $P \leqslant 0.02$. Only one item: 'Number of painful conditions' demonstrated statistically significant DIF $\left(\chi^{2}(1)=10.07, P=0.002\right)$, where this item was more difficult for individuals with non-cervical injuries. However, the DIF contrast, that is the magnitude of the difference in item difficulty between the two groups of persons, was small in magnitude $(0.20$ logits). As it is suggested that DIF contrasts $<0.43$ logits are indicative of a negligible level of DIF, this item was retained. ${ }^{26}$

\section{DISCUSSION}

The present study utilized Rasch analysis to explore the measurement properties of the PMQ in a sample of persons with SCI. Results suggested a subset of 23 items from the PMQ represented a single unidimensional construct. Rasch analysis results revealed that the rating scale and majority of persons (>93\%) and items (20/23) fit the Rasch measurement model. The PMQ demonstrated adequate reliability and functions to separate persons into two strata-those likely to misuse pain medication and those with low liklihood of misusing pain medication. An absence of ceiling effects and minimal $(0.07 \%$ of persons) floor effects were observed when comparing person and item measures. Examination of item measures and thresholds revealed that some PMQ items, particularly those with moderate difficulty, demonstrate measurement overlap; this suggests that item reduction may be possible.

Overall, the PMQ items performed well in the Rasch model. A negligible amount of DIF was observed for one item when comparing individuals with cervical vs non-cervical SCI. Three items demonstrated significant misfit to the model. Those items were:

Item 5-'I [WOULD] mind quitting my current medication and trying a new one if my doctor recommends it'

Item 15-I get pain medication from more than one doctor in order to have enough medication for my pain'

Item 17-'To help me out, family members have obtained pain medications for me from their own doctors'

Two of these items (Items 15 and 17) were found to be the most difficult items to endorse. Post hoc descriptive analyses of these 


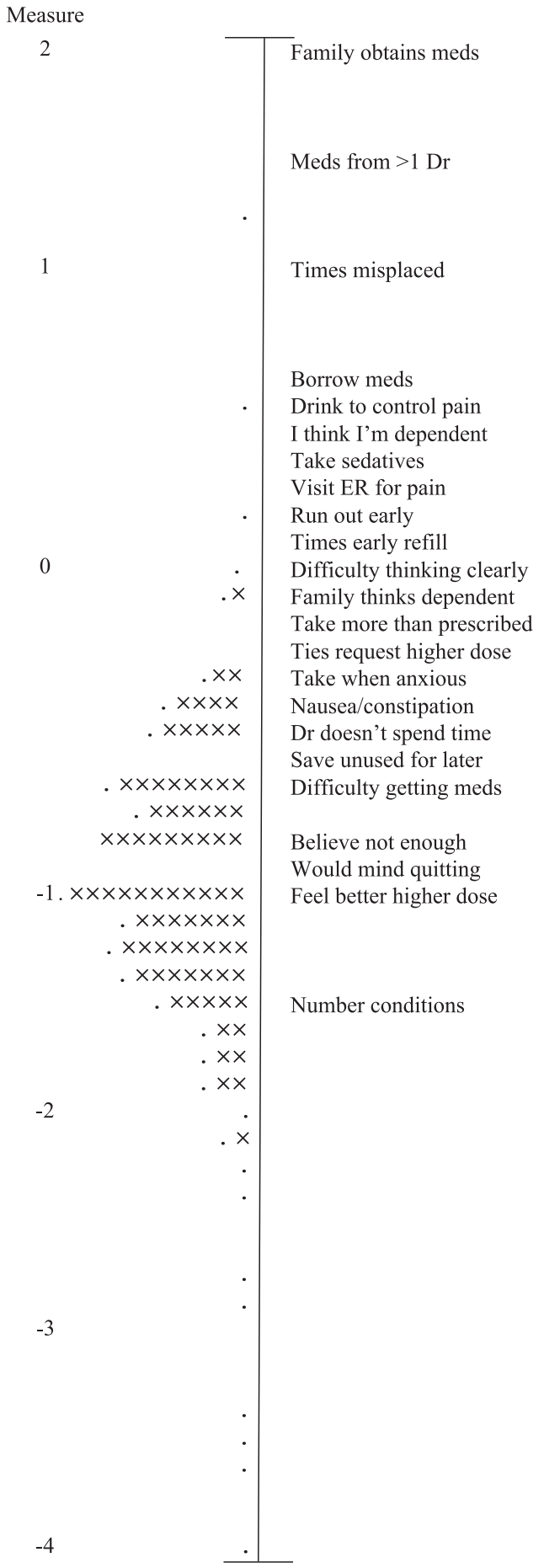

Figure 1 Rasch person-item map depicting relationship of person measures to item difficulties, in logits, on the same interval level scale. Person measures and item difficulties range from low (bottom of figure) to high (top of figure). Each ' $x$ ' represents 8 persons, and each '?' represents 1-7 persons.

variables revealed that $>95 \%$ of respondents answered ' 0 ' to these items, indicating they 'never' engage in those behaviors. It is possible the misfit was a result of invariance in responses to these items such that respondents were either not engaging in these behaviors or did not report engagement in these behaviors. ${ }^{26}$ As a result, these items may be of little value in the measurement of risk of PMM in persons

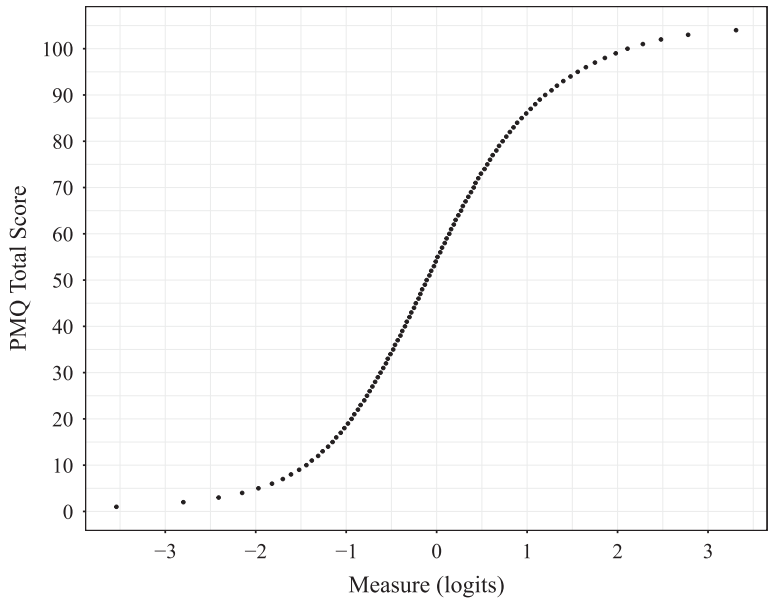

Figure 2 Raw score to measure ogive for complete PMQ. This figure provides a conversion between Rasch-calibrated person measures and item difficulties ( $x$ axis) and PMQ total scores (y axis).

Table 4 Results of DIF analysis for individuals with cervical vs non-cervical SCI

\begin{tabular}{|c|c|c|c|}
\hline Item & $D I F x^{2}$ & P-value & IDIF contrast/ \\
\hline Number conditions ${ }^{a}$ & 10.068 & 0.002 & 0.20 \\
\hline Believe not enough & 2.439 & 0.118 & 0.09 \\
\hline Dr does not spend time & 0.228 & 0.633 & 0.03 \\
\hline Feel better higher dose & 0.000 & 1.000 & 0.00 \\
\hline Difficulty getting meds & 0.000 & 1.000 & 0.00 \\
\hline Would mind quitting & 2.685 & 0.101 & 0.10 \\
\hline Family thinks dependent & 0.145 & 0.704 & 0.02 \\
\hline Take when anxious & 1.699 & 0.192 & 0.10 \\
\hline Drink to control pain & 2.494 & 0.114 & 0.19 \\
\hline Difficulty thinking clearly & 1.224 & 0.269 & 0.10 \\
\hline Visit emergency room for pain & 0.000 & 1.000 & 0.00 \\
\hline Nausea/constipation & 0.386 & 0.535 & 0.03 \\
\hline Borrow meds & 3.470 & 0.063 & 0.24 \\
\hline Meds from $>1 \mathrm{Dr}$ & 1.935 & 0.164 & 0.48 \\
\hline I think I'm dependent & 0.286 & 0.593 & 0.06 \\
\hline Family obtains meds & 1.402 & 0.237 & 0.56 \\
\hline Take more than prescribed & 2.059 & 0.151 & 0.11 \\
\hline Save unused for later & 0.203 & 0.653 & 0.02 \\
\hline Run out early & 0.082 & 0.774 & 0.02 \\
\hline Take sedatives & 0.067 & 0.796 & 0.02 \\
\hline Times request higher dose & 0.000 & 1.000 & 0.00 \\
\hline Times early refill & 1.199 & 0.274 & 0.10 \\
\hline Times misplaced & 1.301 & 0.254 & 0.22 \\
\hline
\end{tabular}

Abbreviations: DIF, differential item functioning; $\mathrm{Dr}$, doctor; $\mathrm{SCl}$, spinal cord injury. Degrees of freedom for all comparisons $=1$.

astatistically significant DIF.

with SCI. However, in other diagnostic populations, these items may be more informative.

Our findings also have important clinical implications. Specifically, results do not support the division of PMQ total scores into tertiles to classify individuals as high, medium or low risk for PMM, as the PMQ distinguishes persons into only two strata. In addition, the commonly utilized cutoff scores ${ }^{6,7}$ may be too low, thus leading to misclassifications of an individual's risk of PMM. Examination of item content in relation to item difficulty hierarchy suggests a cutoff around the items 
'How many times in the past year have you run out of pain medication early and had to request an early refill,' 'At times, I run out of pain medication early and have to call my doctor for refills,' and 'My pain medication makes it hard for me to think clearly sometimes' may be more indicative of PMM, and therefore more clinically meaningful, than the item 'I believe I am [NOT] receiving enough medication to relieve my pain.' These item measures are around 0.05-0.14 logits (Table 3), which corresponds to a PMQ total score of 55-60 (Figure 2).

\section{Limitations and future directions}

The present study has some methodologic considerations worth noting. First, the PMQ was not originally designed to adhere to the Rasch measurement model, which requires a single unidimensional construct and hierarchical item structure. As a result, it is unsurprising that the PMQ failed to meet some Rasch assumptions. Second, the present sample was skewed towards lower likelihood of PMM. It is possible that inclusion of persons with higher likelihood of PMM in the analysis may reveal that the PMQ distinguishes persons into more than two strata. Moving forward, the measurement properties of the PMQ should be studied in individuals with SCI with greater variety in likelihood of PMM to build upon the findings. Third, we utilized a population-based cohort, which is the gold standard for SCI recruitment, since it captures the full range of SCI, including those who may not receive treatment in a rehabilitation or specialty hospital. Therefore, the findings generalize to the full population of SCI, yet, as a whole, it will include a substantially larger portion of individuals who are ambulatory, compared with those treated in traditional specialty hospitals.

\section{CONCLUSION}

Given the exponential increase in the rate of prescription pain medication overdose-related deaths in recent years in the general population, it is critical that clinicians and researchers utilize valid and reliable measures for identifying individuals at elevated risk of PMM. The chronic pain experienced by individuals post-SCI may predispose this population to elevated risk of PMM and subsequent PMM-related comorbidities. Findings of this study suggest 23 of the 26 PMQ items yield valid and reliable estimates of PMM in persons with SCI and functions to distinguish them into two strata-those more likely to misuse pain medication and those with low likelihood of misusing pain medication. Gaining a deeper understanding of the measurement properties of the PMQ is a necessary precursor for widespread population-based studies seeking to elucidate the incidence of PMM in persons with SCI. Future studies that build upon these findings by including individuals with SCI across a broader range of likelihood of PMM are warranted.

\section{CONFLICT OF INTEREST}

The authors declare no conflict of interest.

\section{ACKNOWLEDGEMENTS}

The contents of this publication were developed under a grant from the National Institute on Disability, Independent Living, and Rehabilitation Research (NIDILRR grant number 90RT5003). NIDILRR is a Center within the Administration for Community Living (ACL), Department of Health and Human Services (HHS). The contents of this publication do not necessarily represent the policy of NIDILRR, ACL, HHS, and you should not assume endorsement by the Federal Government. In addition, this publication received support from the South Carolina Spinal Cord Injury Research Fund, grant numbers \#2016 PD-01 and \#09-001.
1 van Gorp S, Kessels AG, Joosten EA, van Kleef M, Patijn J. Pain prevalence and its determinants after spinal cord injury: a systematic review. Eur J Pain 2015; 19: 5-14.

2 Ataoglu E, Tiftik T, Kara M, Tunc H, Ersoz M, Akkus S. Effects of chronic pain on quality of life and depression in patients with spinal cord injury. Spinal Cord 2013; 51: 23-26.

3 Mann R, Schaefer C, Sadosky A, Bergstrom F, Baik R, Parsons B et al. Burden of spinal cord injury-related neuropathic pain in the United States: retrospective chart review and cross-sectional survey. Spinal Cord 2013; 51: 564-570.

4 Cardenas DD, Jensen MP. Treatments for chronic pain in persons with spinal cord injury: a survey study. J Spinal Cord Med 2006; 29: 109-117.

5 Rudd RA, Aleshire N, Zibbell JE, Gladden RM. Increases in drug and opioid overdose deaths-United States, 2000-2014. MMWR Mortal Wkly Rep 2016; 64: 1378-1382.

6 Clark JM, Cao Y, Krause JS. Risk of pain medication misuse after spinal cord injury: The role of substance use, personality, and depression. J Pain 2017; 18: 166-177.

7 Krause JS, Clark JM, Saunders LL. Pain medication misuse among participants with spinal cord injury. Spinal Cord 2015; 53: 630-635.

8 Saunders LL, Dipiro N, Krause JS, Brotherton S, Kraft S. Risk of fall related injuries among ambulatory participants with spinal cord injury. Top Spinal Cord Inj Rehabil 2013; 19: 259-266.

9 Chou R, Turner JA, Devine EB, Hansen RN, Sullivan SD, Blazina I et al. The effectiveness and risks of long-term opioid therapy for chronic pain: a systematic review for a National Institutes of Health Pathways to Prevention Workshop. Ann Intern Med 2015; 162: 276-286.

10 Thietje R, Pouw MH, Schulz AP, Kienast B, Hirschfeld S. Mortality in patients with traumatic spinal cord injury: descriptive analysis of 62 deceased subjects. J Spinal Cord Med 2011; 34: 482-487.

11 Carbone LD, Chin AS, Burns SP, Svircev JN, Hoenig H, Heggeness M et al. Mortality after lower extremity fractures in men with spinal cord injury. J Bone Miner Res 2014; 29: 432-439.

12 Adams LL, Gatchel RJ, Robinson RC, Polatin P, Gajraj N, Deschner M et al. Development of a self-report screening instrument for assessing potential opioid medication misuse in chronic pain patients. J Pain Symptom Manage 2004; 27: 440-459.

13 Dowling LS, Gatchel RJ, Adams LL, Stowell AW, Bernstein D. An evaluation of the predictive validity of the Pain Medication Questionnaire with a heterogeneous group of patients with chronic pain. J Opioid Manag 2007; 3: 257-266.

14 Holmes CP, Gatchel RJ, Adams LL, Stowell AW, Hatten A, Noe C et al. An opioid screening instrument: long-term evaluation of the utility of the Pain Medication Questionnaire. Pain Pract 2006; 6: 74-88.

15 Buelow AK, Haggard R, Gatchel RJ. Additional validation of the pain medication questionnaire in a heterogeneous sample of chronic pain patients. Pain Pract 2009; 9: 428-434.

16 Wright BD. Additivity in psychological measurement. In: Roskam EE (ed.). Measurement and Personality Assessment. Elsevier Science Publishers: North-Holland. 1985, pp 101-111.

17 Hojsted J, Nielsen PR, Kendall S, Frich L, Sjogren P. Validation and usefulness of the Danish version of the Pain Medication Questionnaire in opioid-treated chronic pain patients. Acta Anaesthesiol Scand 2011; 55: 1231-1238.

18 Hobart J, Cano S. Improving the evaluation of therapeutic interventions in multiple sclerosis: the role of new psychometric methods. Health Technol Assess 2009; 13: iii, $1-177$.

19 Granger C. Rasch analysis is important to understand and use for measurement. Rasch Meas Transac 2008; 21: 1122-1123.

20 Wright BD, Masters GN. Number of person or item strata. Rasch Meas Transac 2002; 16: 888 .

21 Prieto L, Alonso J, Lamarca R. Classical test theory versus Rasch analysis for quality of life questionnaire reduction. Health Qual Life Outcomes 2003; 1: 27.

22 Varma A, Hill EG, Nicholas J, Selassie A. Predictors of early mortality after traumatic spinal cord injury: a population-based study. Spine 2010; 35: 778-783.

23 Revelle W. Package "psych" 2016. Available from https://cran.r-project.org/web/ packages/psych/psych.pdf.

24 Holgado-Tello FP, Chacón-Moscoso S, Barbero-Garcia I, Vila-Abad E. Polychoric versus Pearson correlations in exploratory and confirmatory factor analysis of ordinal variables. Qual Quan 2010; 44: 153-166.

25 Tabachnick B, Fidell L. Using Multivariate Statistics 6th edn. Pearson: Harlow, 2014.

26 Linacre JM. Winsteps (R) Rasch measurement computer program: User's guide 2016. Available from: http://www.winsteps.com/winman/principalcomponents.htm.

27 Smith EV Jr. Detecting and evaluating the impact of multidimensionality using item fit statistics and principal component analysis of residuals. J App/ Meas 2002; 3: 205-231.

28 Linacre JM. Winsteps (R) Rasch measurement computer program 2016. Available from: winsteps.com.

29 Linacre JM. Optimizing rating scale category effectiveness. J Appl Meas 2002; 3: 85-106.

30 Wright BD, Linacre JM. Reasonable mean-square fit values. Rasch Measurement Transactions 1994; 8: 370.

31 Siddall PJ, Taylor DA, McClelland JM, Rutkowski SB, Cousins MJ. Pain report and the relationship of pain to physical factors in the first 6 months following spinal cord injury. Pain 1999; 81: 187-197.

32 Finnerup NB, Norrbrink C, Trok K, Piehl F, Johannesen IL, Sorensen JC et al. Phenotypes and predictors of pain following traumatic spinal cord injury: a prospective study. J Pain 2014; 15: 40-48. 